Probability, Networks and Algorithms 
CWI is the National Research Institute for Mathematics and Computer Science. It is sponsored by the Netherlands Organization for Scientific Research (NWO).

$\mathrm{CWI}$ is a founding member of ERCIM, the European Research Consortium for Informatics and Mathematics.

CWI's research has a theme-oriented structure and is grouped into four clusters. Listed below are the names of the clusters and in parentheses their acronyms.

\section{Probability, Networks and Algorithms (PNA)}

Software Engineering (SEN)

Modelling, Analysis and Simulation (MAS)

Information Systems (INS)

Copyright (C) 2003, Stichting Centrum voor Wiskunde en Informatica

P.O. Box 94079, 1090 GB Amsterdam (NL)

Kruislaan 413, 1098 SJ Amsterdam (NL)

Telephone +31205929333

Telefax +31205924199

ISSN 1386-3711 


\title{
Bandwidth Sharing with Heterogeneous Service Requirements
}

\begin{abstract}
We consider a system with two heterogeneous traffic classes. The users from both classes randomly generate service requests, one class having light-tailed properties, the other one exhibiting heavy-tailed characteristics. The heterogeneity in service requirements reflects the extreme variability in flow sizes observed in the Internet, with a vast majority of small transfers ('mice') and a limited number of exceptionally large flows ('elephants'). The active traffic flows share the available bandwidth in a Processor-Sharing (PS) fashion. The PS discipline has emerged as a natural paradigm for modeling the flow-level performance of bandwidth-sharing protocols like TCP. The number of simultaneously active traffic flows is limited by a threshold on the maximum system occupancy. We obtain the exact asymptotics of the transfer delays incurred by the users from the light-tailed class. The results show that the threshold mechanism significantly reduces the detrimental performance impact of the heavy-tailed class.
\end{abstract}

2000 Mathematics Subject Classification: 60K25 (primary), 68M20, 90B18, 90B22 (secondary)

Keywords and Phrases: delay asymptotics, heterogeneous traffic, light tails and heavy tails, processor sharing, admission control.

Note: Work partially carried out under the CWI project P1201. 


\title{
Bandwidth Sharing with Heterogeneous Service Requirements
}

\author{
Sem Borst ${ }^{\dagger, \star \star \ddagger}$ Rudesindo Núñez-Queija ${ }^{\dagger, \star}$, Bert Zwart ${ }^{\dagger, \star}$ \\ ${ }^{\dagger} \mathrm{CWI}$ \\ P.O. Box 94079, 1090 GB Amsterdam, The Netherlands \\ ${ }^{\star}$ Department of Mathematics \& Computer Science \\ Eindhoven University of Technology \\ P.O. Box 513, 5600 MB Eindhoven, The Netherlands \\ $\ddagger$ Bell Laboratories, Lucent Technologies \\ P.O. Box 636, Murray Hill, NJ 07974, USA
}

\begin{abstract}
We consider a system with two heterogeneous traffic classes. The users from both classes randomly generate service requests, one class having light-tailed properties, the other one exhibiting heavy-tailed characteristics. The heterogeneity in service requirements reflects the extreme variability in flow sizes observed in the Internet, with a vast majority of small transfers ('mice') and a limited number of exceptionally large flows ('elephants'). The active traffic flows share the available bandwidth in a Processor-Sharing (PS) fashion. The PS discipline has emerged as a natural paradigm for modeling the flow-level performance of bandwidth-sharing protocols like TCP. The number of simultaneously active traffic flows is limited by a threshold on the maximum system occupancy. We obtain the exact asymptotics of the transfer delays incurred by the users from the light-tailed class. The results show that the threshold mechanism significantly reduces the detrimental performance impact of the heavy-tailed class.
\end{abstract}

2000 Mathematics Subject Classification: 60K25 (primary), 68M20, 90B18, 90B22 (secondary).

Keywords and Phrases: delay asymptotics, heterogeneous traffic, light tails and heavy tails, processor sharing, admission control.

Note: Work partially carried out under the CWI project P1201.

\section{Introduction}

Over the past decade, TCP has gained ubiquity as the predominant congestion control mechanism for elastic traffic in the Internet. Wide-ranging efforts have been made to obtain a better understanding of the performance characteristics of TCP. One thread of research has been concerned with estimating the throughput achieved by long-lived TCP flows for given round-trip delay and packet loss statistics, see for instance $[1,8,16]$. 
A further body of literature has focused on describing the behavior of dynamically interacting TCP flows. On the latter front, the Processor-Sharing (PS) discipline has been widely adopted as a convenient modeling abstraction for the bandwidth sharing among competing TCP users $[2,12,13]$. In addition, PS models have proven useful in evaluating the flow-level performance in emerging wireless data networks.

Independently, extensive measurement studies have indicated that file sizes in the Internet, and hence the data volumes of TCP transfers, commonly exhibit heavy-tailed features. These observations have triggered a huge interest in the delay characteristics of PS queues with heavy-tailed service requirements. Zwart \& Boxma [19] obtained the delay asymptotics for regularly varying service requirements using transform techniques. They proved that the tail of the delay distribution is asymptotically equivalent to that of the service time distribution, up to a constant factor. Subsequently, Zwart [18] generalized the result to multi-class PS queues. Using a proof based on conditional moments, Núñez-Queija [13, 14] extended the tail equivalence result to PS models with a time-varying service capacity and intermediately regularly varying service requirements. Jelenković \& Momčilović [9] used a probabilistic proof method to generalize the result to a larger subclass of subexponential distributions with a so-called square-root insensitivity property. The latter class includes Weibull distributions with an index parameter smaller than $1 / 2$. They further showed that the result is sharp, in the sense that the tail equivalence does not hold for Weibull distributions with a larger index parameter. Recently, Boyer et al. [4] obtained a tail equivalence result for heavy-tailed PS queues with impatient users and admission control. In contrast to the remarkably explicit results for heavy-tailed PS queues, the delay asymptotics for light-tailed service requirements have remained largely intractable. The sojourn time asymptotics for exponentially distributed service requirements were obtained via a useful equivalence relation with Random-Order-of-Service queues [3, 7]. These asymptotics turn out to have a rather intriguing transcendental structure. To the best of our knowledge, there are no expressions available for general light-tailed distributions.

In the present paper, we consider a PS system with two heterogeneous traffic classes, one class having light-tailed features, the other one exhibiting heavy-tailed characteristics. The disparity in service requirements reflects the extreme diversity in flow sizes observed in the Internet, with the bulk of the flows comprising just a couple of packets ('mice') and a tiny fraction of huge transfers ('elephants'). We assume that the number of simultaneously active traffic flows is limited by a threshold on the maximum system occupancy. We derive the exact delay asymptotics for the light-tailed class. Although the PS paradigm may not be entirely justified for short flows, inspection of the proofs suggests that this assumption is actually not that crucial for the asymptotic results to hold.

Our main results (Theorems 3.1 and 4.1) provide two important qualitative insights. First of all, they show that the PS discipline does not prevent the light-tailed class from being affected by the heavy-tailed class, which indicates that the "positive" results in the abovementioned papers should be treated with caution. Secondly, our results demonstrate that the negative performance impact of the heavy-tailed class can be reduced by limiting the number of simultaneously active users in the system. These findings reinforce some of the arguments made in $[4,11]$ in favor of admission control for TCP flows. It is worth observing that in practice TCP time-out effects and user impatience may indirectly induce a limit on the maximum number of active flows (although the actual mechanics are quite different). It is further noteworthy that in wireless data networks for instance there is an intrinsic limit on the number of active users due to various system constraints, even in the 
absence of any explicit admission control.

The remainder of the paper is organized as follows. In Section 2, we present a detailed model description and collect a few preliminary results. In Section 3, we derive the exact delay asymptotics for the users from the light-tailed class. In Section 4 , we examine the case with no admission control. The paper concludes with a few final remarks in Section 5.

\section{Model description and preliminary results}

Consider a system with two heterogeneous traffic classes. Class- $i$ users arrive as a Poisson process of rate $\lambda_{i}$ and require an amount of service $\mathbf{B}_{i}$ with mean $\beta_{i}, i=1,2$. Define $\rho_{i}:=\lambda_{i} \beta_{i}$ as the traffic intensity of class $i$. Denote by $\lambda:=\lambda_{1}+\lambda_{2}$ the total arrival rate and by $\rho:=\rho_{1}+\rho_{2}$ the total traffic intensity.

We assume that the class-1 service requirements have light-tailed properties, whereas the class-2 service requirements have heavy-tailed characteristics. Specifically, the class-2 service requirement distribution is regularly varying of index $-\nu_{2}<-1$, i.e.,

$$
\operatorname{Pr}\left\{\mathbf{B}_{2}>x\right\} \sim \frac{C_{2}}{-\Gamma\left(1-\nu_{2}\right)} x^{-\nu_{2}} l_{2}(x),
$$

with $C_{2}>0$ some constant and $l_{2}(\cdot)$ a slowly varying function. Here, as well as in the remainder of the paper, we use the notational convention $f(x) \sim g(x)$ to indicate that $\lim _{x \rightarrow \infty} f(x) / g(x)=1$. For any non-negative random variable $\mathbf{Y}$ with $\mathbb{E} \mathbf{Y}<\infty$, denote by $\mathbf{Y}^{r}$ a random variable representing the residual lifetime of $\mathbf{Y}$, i.e., $\operatorname{Pr}\left\{\mathbf{Y}^{r}>x\right\}=$ $\frac{1}{\mathbb{E} \mathbf{Y}} \int_{0}^{x} \operatorname{Pr}\{\mathbf{Y}>u\} \mathrm{d} u$. In particular, we have

$$
\operatorname{Pr}\left\{\mathbf{B}_{2}^{r}>x\right\} \sim \frac{C_{2}}{\beta_{2} \Gamma\left(2-\nu_{2}\right)} x^{1-\nu_{2}} l_{2}(x) .
$$

We assume that the class- 1 service requirement distribution has the following representation:

$$
\operatorname{Pr}\left\{\mathbf{B}_{1}>x\right\}=q_{1}(x) \mathrm{e}^{-x^{\eta_{1}}},
$$

with $q_{1}(x)$ is a regularly varying function and $\eta_{1}>0$. The above representation allows not only light-tailed random variables, but also Weibullian random variables, which are subexponential.

The next lemma will be useful.

Lemma 2.1 There exists a function $D(x)$, with $D(x)=\mathrm{o}(x)$, such that for any $K$,

$$
\frac{\operatorname{Pr}\left\{\mathbf{B}_{1}>x+D(x)\right\}}{\operatorname{Pr}\left\{\mathbf{B}_{1}>x\right\}}=\mathrm{o}\left(\left(\operatorname{Pr}\left\{\mathbf{B}_{2}^{r}>\frac{x}{K}\right\}\right)^{K-1}\right),
$$

as $x \rightarrow \infty$. In particular, we can choose $D(x)=x^{\gamma}$ for any $\gamma \in\left(1-\eta_{1}, 1\right)$.

\section{Proof}

Write

$$
\frac{\operatorname{Pr}\left\{\mathbf{B}_{1}>x+D(x)\right\}}{\operatorname{Pr}\left\{\mathbf{B}_{1}>x\right\}}=\frac{q_{1}(x+D(x))}{q_{1}(x)} \mathrm{e}^{-\left((x+D(x))^{\eta_{1}}-x^{\eta_{1}}\right)},
$$


and observe that, for any $\eta_{1}>0$,

$$
(x+D(x))^{\eta_{1}}-x^{\eta_{1}}=\eta_{1} \int_{x}^{x+D(x)} y^{\eta_{1}-1} \mathrm{~d} y \geq \eta_{1} \min \left(x^{\eta_{1}-1},(x+D(x))^{\eta_{1}-1}\right) D(x) .
$$

Choosing $D(x)=x^{\gamma}$, with $\gamma \in\left(1-\eta_{1}, 1\right)$, we have $(x+D(x))^{\eta_{1}-1} D(x) \sim x^{\eta_{1}-1} D(x) \sim$ $x^{\eta_{1}-1+\gamma}$. This proves the lemma since $\eta_{1}-1+\gamma>0$ and $\left(\operatorname{Pr}\left\{\mathbf{B}_{2}^{r}>\frac{x}{K}\right\}\right)^{K-1}$ is regularly varying.

The users in the system are served according to the Processor-Sharing (PS) discipline, i.e., the total service rate is equally shared among all the users present. Thus, when there are $n$ users present, each of them receives service at rate $1 / n$. The admission of users is subject to a threshold $K$ on the maximum occupancy of the system. Users that arrive when there are already $K$ users present, are blocked and lost from the system.

Denote by $\mathbf{V}_{i}$ the sojourn time of an arbitrary class- $i$ user. By convention, the sojourn time of blocked users is considered to be zero.

Denote by $\mathbf{N}$ the total number of users present at an arbitrary epoch. Given that $\mathbf{N}=n$, let $\mathbf{U}_{1}, \ldots, \mathbf{U}_{n}$ and $\mathbf{X}_{1}, \ldots, \mathbf{X}_{n}$ be the class indices and the remaining service requirements of the users present.

According to Cohen [5], Kelly [10],

$$
\begin{aligned}
& \operatorname{Pr}\left\{\mathbf{N}=n ;\left(\mathbf{U}_{1}, \ldots, \mathbf{U}_{n}\right)=\left(u_{1}, \ldots, u_{n}\right) ;\left(\mathbf{X}_{1}, \ldots, \mathbf{X}_{n}\right)>\left(x_{1}, \ldots, x_{n}\right)\right\}= \\
& \frac{(1-\rho) \rho^{n}}{1-\rho^{K+1}} \prod_{k=1}^{n} \frac{\rho_{u_{k}}}{\rho} \operatorname{Pr}\left\{\mathbf{B}_{u_{k}}^{r}>x_{k}\right\},
\end{aligned}
$$

so that

$$
\begin{aligned}
& \operatorname{Pr}\left\{\mathbf{N}=n ;\left(\mathbf{X}_{1}, \ldots, \mathbf{X}_{n}\right)>\left(x_{1}, \ldots, x_{n}\right)\right\}= \\
& \frac{(1-\rho) \rho^{n}}{1-\rho^{K+1}} \prod_{k=1}^{n}\left(\frac{\rho_{1}}{\rho} \operatorname{Pr}\left\{\mathbf{B}_{1}^{r}>x_{k}\right\}+\frac{\rho_{2}}{\rho} \operatorname{Pr}\left\{\mathbf{B}_{2}^{r}>x_{k}\right\}\right) .
\end{aligned}
$$

\section{Delay asymptotics with admission control}

The next theorem provides the main result of the paper.

Theorem 3.1 For any fixed value of $K<\infty$,

$$
\operatorname{Pr}\left\{\mathbf{V}_{1}>x\right\} \sim \operatorname{Pr}\left\{\mathbf{B}_{1}>\frac{x}{K}\right\} \frac{(1-\rho) \rho_{2}^{K-1}}{1-\rho^{K+1}}\left(\operatorname{Pr}\left\{\mathbf{B}_{2}^{r}>\frac{x}{K}\right\}\right)^{K-1} .
$$

Before proving the above theorem, we first present a heuristic interpretation. Consider a tagged class-1 user arriving to the system at time 0. Observe that the right-hand side of (3) represents the probability of the following two events occurring: (i) the service requirement of the tagged user is at least $x / K$; (ii) when the tagged user arrives, there are exactly $K-1$ class- 2 users present, each of which has a remaining service requirement of at least $x / K$. Note that these two events combined imply that the sojourn time of the tagged user exceeds the value $x$. The above theorem indicates that these two events are 
in fact the only plausible scenario for the sojourn time to exceed the value $x$. This may be intuitively explained as follows. Observe that the threshold mechanism ensures that each admitted user receives service at least at rate $1 / K$. Hence, for the sojourn time to exceed the value $x$, the service requirement of the tagged user must indeed be at least $x / K$. In addition, at least one of the following two scenarios must occur in order for the sojourn time to exceed the value $x$ : (I) the service requirement of the tagged user is at least $x / K+D(x)$; (II) the amount of service received by the tagged user during the time interval $[0, x]$ is at most $x / K+D(x)$. By Lemma 2.1 , the first scenario is highly unlikely if we choose $D(x)=x^{\gamma}$ with $\gamma>1-\eta_{1}$. The proof of Lemma 3.1 below shows that the second scenario is extremely implausible, unless there are exactly $K-1$ class- 2 users present when the tagged user arrives, each of which has a remaining service requirement of at least $x / K$. Thus the second scenario asymptotically coincides with the occurrence of the event (ii) described above.

Lemma 3.1 Let $S(0, t)$ be the amount of service received during the interval $[0, t]$ by a user which arrives to the system at time 0 and stays in the system permanently. For any non-negative function $D(\cdot)$ with $D(x)=\mathrm{o}(x)$ as $x \rightarrow \infty$ and fixed value of $K<\infty$,

$$
\operatorname{Pr}\left\{S(0, x) \leq \frac{x}{K}+D(x)\right\} \sim \frac{(1-\rho) \rho_{2}^{K-1}}{1-\rho^{K+1}}\left(\operatorname{Pr}\left\{\mathbf{B}_{2}^{r}>\frac{x}{K}\right\}\right)^{K-1} .
$$

Note that Lemma 3.1 may be rephrased in terms of conditional delay asymptotics as follows. Let $B$ and $V$ be the service requirement and sojourn time of a tagged user arriving to the system at time 0 . Then

$$
\operatorname{Pr}\left\{V>x \mid B=\frac{x}{K}+D(x)\right\} \sim \frac{(1-\rho) \rho_{2}^{K-1}}{1-\rho^{K+1}}\left(\operatorname{Pr}\left\{\mathbf{B}_{2}^{r}>\frac{x}{K}\right\}\right)^{K-1} .
$$

For completeness, we also give the delay asymptotics for class- 2 users. It follows from results in [4] that

$$
\operatorname{Pr}\left\{\mathbf{V}_{2}>x\right\} \sim \operatorname{Pr}\left\{\mathbf{B}_{2}>\gamma_{K} x\right\},
$$

where $\gamma_{K}$ denotes the mean service rate of a fictitious permanent user in a system with admission threshold $K$. Note that $\gamma_{K}=1-\rho \alpha_{K}$ with $\alpha_{K}:=\frac{1-\rho^{K}}{1-\rho^{K+1}}$ the probability that an arriving user finds less than $K$ users present and is admitted into the system. In particular, $\gamma_{\infty}=1-\rho \alpha_{\infty}=1-\rho$. Combining the above result with Theorem 3.1, we find

$$
\operatorname{Pr}\{V>x\}=\frac{\lambda_{1}}{\lambda} \operatorname{Pr}\left\{\mathbf{V}_{1}>x\right\}+\frac{\lambda_{2}}{\lambda} \operatorname{Pr}\left\{\mathbf{V}_{2}>x\right\} \sim \frac{\lambda_{2}}{\lambda} \operatorname{Pr}\left\{\mathbf{B}_{2}>\gamma_{K} x\right\} .
$$

\section{Proof of Theorem 3.1}

Lower bound: Using Lemma 3.1 with $D(x)=0$, we obtain, for $\delta>0$,

$$
\begin{aligned}
\operatorname{Pr}\left\{\mathbf{V}_{1}>x\right\} & \geq \operatorname{Pr}\left\{\mathbf{B}_{1}>\frac{x}{K}\right\} \operatorname{Pr}\left\{S(0, x) \leq \frac{x}{K}\right\} \\
& \sim \operatorname{Pr}\left\{\mathbf{B}_{1}>\frac{x}{K}\right\} \frac{(1-\rho) \rho_{2}^{K-1}}{1-\rho^{K+1}}\left(\operatorname{Pr}\left\{\mathbf{B}_{2}^{r}>\frac{x}{K}\right\}\right)^{K-1} .
\end{aligned}
$$

Upper bound: Let $B$ and $V$ be the service requirement and sojourn time of a tagged class-1 user arriving to the system at time 0 , respectively. Let $D(x)$ be as in Lemmas 2.1 and 3.1. 
Then,

$$
\begin{aligned}
\operatorname{Pr}\left\{\mathbf{V}_{1}>x\right\} & =\operatorname{Pr}\left\{V>x \mid B \leq \frac{x}{K}\right\} \operatorname{Pr}\left\{B \leq \frac{x}{K}\right\} \\
& +\operatorname{Pr}\left\{V>x \mid B>\frac{x}{K}+D(x)\right\} \operatorname{Pr}\left\{B>\frac{x}{K}+D(x)\right\} \\
& +\operatorname{Pr}\left\{V>x \mid \frac{x}{K}<B \leq \frac{x}{K}+D(x)\right\} \operatorname{Pr}\left\{\frac{x}{K}<B \leq \frac{x}{K}+D(x)\right\} \\
& \leq 0+\operatorname{Pr}\left\{S(0, x) \leq \frac{x}{K}+D(x)\right\} \operatorname{Pr}\left\{\mathbf{B}_{1}>\frac{x}{K}\right\}+\operatorname{Pr}\left\{\mathbf{B}_{1}>\frac{x}{K}+D(x)\right\} \\
& \sim \operatorname{Pr}\left\{\mathbf{B}_{1}>\frac{x}{K}\right\}\left(\frac{(1-\rho) \rho_{2}^{K-1}}{1-\rho^{K+1}}\left(\operatorname{Pr}\left\{\mathbf{B}_{2}^{r}>\frac{x}{K}\right\}\right)^{K-1}+\frac{\operatorname{Pr}\left\{\mathbf{B}_{1}>\frac{x}{K}+D(x)\right\}}{\operatorname{Pr}\left\{\mathbf{B}_{1}>\frac{x}{K}\right\}}\right) \\
& \sim \operatorname{Pr}\left\{\mathbf{B}_{1}>\frac{x}{K}\right\} \frac{(1-\rho) \rho_{2}^{K-1}}{1-\rho^{K+1}}\left(\operatorname{Pr}\left\{\mathbf{B}_{2}^{r}>\frac{x}{K}\right\}\right)^{K-1}
\end{aligned}
$$

which completes the proof.

\section{Proof of Lemma 3.1}

Lower bound: Using (2), we obtain

$$
\begin{aligned}
\operatorname{Pr}\left\{S(0, x) \leq \frac{x}{K}+D(x)\right\} & \geq \operatorname{Pr}\left\{S(0, x)=\frac{x}{K}\right\} \\
& =\operatorname{Pr}\left\{\mathbf{N}=K-1 ;\left(\mathbf{X}_{1}, \ldots, \mathbf{X}_{K-1}\right)>\left(\frac{x}{K}, \frac{x}{K}, \ldots, \frac{x}{K}\right)\right\} \\
& =\frac{1-\rho}{1-\rho^{K+1}}\left(\rho_{1} \operatorname{Pr}\left\{\mathbf{B}_{1}^{r}>\frac{x}{K}\right\}+\rho_{2} \operatorname{Pr}\left\{\mathbf{B}_{2}^{r}>\frac{x}{K}\right\}\right)^{K-1} \\
& \geq \frac{(1-\rho) \rho_{2}^{K-1}}{1-\rho^{K+1}}\left(\operatorname{Pr}\left\{\mathbf{B}_{2}^{r}>\frac{x}{K}\right\}\right)^{K-1} .
\end{aligned}
$$

Upper bound: Let $T(s, t)$ be the amount of time during the interval $[s, t]$ that there are $K-2$ or less users in the system, other than the permanent user. Note that the permanent user receives service at rate at least $1 /(K-1)$ when there are $K-2$ or less other users in the system. Thus,

$$
S(0, x) \geq(x-T(0, x)) \frac{1}{K}+T(0, x) \frac{1}{K-1}=\frac{x}{K}+T(0, x)\left(\frac{1}{K-1}-\frac{1}{K}\right),
$$

which implies

$$
\operatorname{Pr}\left\{S(0, x) \leq \frac{x}{K}+D(x)\right\} \leq \operatorname{Pr}\{T(0, x) \leq E(x)\},
$$

with $E(x):=D(x) /\left(\frac{1}{K-1}-\frac{1}{K}\right)$.

In order to evaluate the latter probability, we will distinguish between users with 'small' and 'large' service requirements. In particular, we will find it useful to consider the number of users whose (remaining) service requirement is larger than $\epsilon x$ for some small but fixed value of $\epsilon$. Let $N_{>z}(s)$ be the number of users present at time $s$ whose remaining service requirement is larger than $z$. Let $M_{>z}(s, t)$ be the number of new users admitted into the system during the time interval $[s, t]$ whose service requirement is larger than $z$. Denote $N_{>z}(s, t):=N_{>z}(s)+M_{>z}(s, t)$. Then, for $\delta \in(0,1)$, 


$$
\begin{aligned}
& \operatorname{Pr}\{T(0, x) \leq E(x)\} \\
= & \operatorname{Pr}\left\{T(0, x) \leq E(x) ; N_{>\epsilon x}(0, x) \leq K-1 ; N_{>\epsilon x}(0, \delta x)=K-1 ; N_{>\epsilon x}((1-\delta) x, x)=K-1\right\} \\
+ & \operatorname{Pr}\left\{T(0, x) \leq E(x) ; N_{>\epsilon x}(0, x) \leq K-1 ; N_{>\epsilon x}(0, \delta x)=K-1 ; N_{>\epsilon x}((1-\delta) x, x) \leq K-2\right\} \\
+ & \operatorname{Pr}\left\{T(0, x) \leq E(x) ; N_{>\epsilon x}(0, x) \leq K-1 ; N_{>\epsilon x}(0, \delta x) \leq K-2\right\} \\
+ & \operatorname{Pr}\left\{T(0, x) \leq E(x) ; N_{>\epsilon x}(0, x) \geq K\right\},
\end{aligned}
$$

which is obviously upper bounded by

$$
\begin{aligned}
& \operatorname{Pr}\left\{N_{>\epsilon x}(0, x)=K-1 ; N_{>\epsilon x}(0, \delta x)=K-1 ; N_{>\epsilon x}((1-\delta) x, x)=K-1\right\} \\
+ & \operatorname{Pr}\left\{T((1-\delta) x, x) \leq E(x) ; N_{>\epsilon x}((1-\delta) x, x) \leq K-2\right\} \\
+ & \operatorname{Pr}\left\{T(0, \delta x) \leq E(x) ; N_{>\epsilon x}(0, \delta x) \leq K-2\right\} \\
+ & \operatorname{Pr}\left\{N_{>\epsilon x}(0, x) \geq K\right\} \\
= & \mathrm{I}+\mathrm{II}+\mathrm{III}+\mathrm{IV} .
\end{aligned}
$$

We first consider term I. For the relevant event to occur, there must be $K-1$ users in the system at time $s=\delta x$ all of which are still in the system at time $t=(1-\delta) x$. Thus, each of these users must have a remaining service requirement of at least $(1-2 \delta) x / K$. In fact, $K-1-n$ of these users must already have been in the system at time 0 with a remaining service requirement of at least $(1-\delta) x / K$, or there must have been at least $n$ users arriving during the time interval $[0, \delta x]$ with a service requirement exceeding $(1-2 \delta) x / K$, $0 \leq n \leq K-1$. Conditioning upon the number of large users in the system at time 0 and using (2), we obtain

$$
\begin{aligned}
\mathrm{I} & \leq \frac{1-\rho}{1-\rho^{K+1}} \sum_{n=0}^{K-1}\left(\rho_{1} \operatorname{Pr}\left\{\mathbf{B}_{1}^{r}>(1-\delta) \frac{x}{K}\right\}+\rho_{2} \operatorname{Pr}\left\{\mathbf{B}_{2}^{r}>(1-\delta) \frac{x}{K}\right\}\right)^{K-1-n} \\
& \times \frac{1}{n !}\left(\delta x\left(\lambda_{1} \operatorname{Pr}\left\{\mathbf{B}_{1}>(1-2 \delta) \frac{x}{K}\right\}+\lambda_{2} \operatorname{Pr}\left\{\mathbf{B}_{2}>(1-2 \delta) \frac{x}{K}\right\}\right)\right)^{n} \\
& \sim \frac{1-\rho}{1-\rho^{K+1}}\left(\rho_{1} \operatorname{Pr}\left\{\mathbf{B}_{1}^{r}>(1-\delta) \frac{x}{K}\right\}+\rho_{2} \operatorname{Pr}\left\{\mathbf{B}_{2}^{r}>(1-\delta) \frac{x}{K}\right\}\right)^{K-1} \\
& +\frac{1-\rho}{1-\rho^{K+1}} \sum_{n=1}^{K-1}\left(\rho_{2} \operatorname{Pr}\left\{\mathbf{B}_{2}^{r}>(1-\delta) \frac{x}{K}\right\}\right)^{K-1-n} \frac{1}{n !}\left(\delta x \lambda_{2} \operatorname{Pr}\left\{\mathbf{B}_{2}>(1-2 \delta) \frac{x}{K}\right\}\right)^{n} .
\end{aligned}
$$

Using Lemmas 3.2 and 3.3 below and taking $E(x)=x^{\gamma}, \gamma \in(0,1)$, we obtain

$$
\mathrm{II}+\mathrm{III}+\mathrm{IV}=\mathrm{o}\left(\left(\operatorname{Pr}\left\{\mathbf{B}_{2}^{r}>\frac{x}{K}\right\}\right)^{K-1}\right)
$$

as $x \rightarrow \infty$. Using the fact that $\operatorname{Pr}\left\{\mathbf{B}_{2}^{r}>x\right\}$ is regularly varying and, hence, $\operatorname{Pr}\left\{\mathbf{B}_{2}^{r}>x\right\}=$ $\mathrm{O}\left(x \operatorname{Pr}\left\{\mathbf{B}_{2}>x\right\}\right)$ as $x \rightarrow \infty$, it follows that all terms in the sum in I can be neglected by passing $\delta \rightarrow 0$, which completes the proof.

We conclude this section with two technical lemmas that were used in the proof of Lemma 3.1. The proofs of Lemmas 3.2 and 3.3 may be found in Appendices A and B, respectively.

Lemma 3.2 For any $\epsilon>0, \operatorname{Pr}\left\{N_{>\epsilon x}(0, x) \geq K\right\}=\mathrm{o}\left(\left(\operatorname{Pr}\left\{\mathbf{B}_{2}^{r}>x\right\}\right)^{K-1}\right)$ as $x \rightarrow \infty$. 
Lemma 3.3 Let $\delta \in(0,1), \zeta>0$ and $E(x)=\mathrm{o}(x)$. Then, for any $\alpha \in[0,1-\delta]$ there exists an $\epsilon^{*}>0$ such that for all $\epsilon \in\left(0, \epsilon^{*}\right]$,

$$
\operatorname{Pr}\left\{T(\alpha x,(\alpha+\delta) x) \leq E(x) ; N_{>\epsilon x}(\alpha x,(\alpha+\delta) x) \leq K-2\right\}=\mathrm{o}\left(x^{-\zeta}\right),
$$

as $x \rightarrow \infty$.

\section{No admission control}

When $K<\infty$, the tail of $\operatorname{Pr}\left\{\mathbf{V}_{1}>x\right\}$ is not heavier than that of $\operatorname{Pr}\left\{\mathbf{B}_{1}>\frac{x}{K}\right\}$. In particular, if the class-1 service requirement distribution has an exponential tail, then so has the sojourn time distribution. In the present section, we show that this property fails to hold when $K=\infty$. We restrict our attention to the case where the class- 1 service requirement distribution is Weibullian, but the result may be shown to hold under more general distributional assumptions. The derivation of an upper bound is left as a topic for further study.

Theorem 4.1 When $\operatorname{Pr}\left\{\mathbf{B}_{1}>x\right\}=\mathrm{e}^{-\mu_{1} x^{\eta_{1}}}$ and $K=\infty$, i.e., no admission control, the sojourn time distribution of class-1 users satisfies, for fixed $\delta>0$ and $x \rightarrow \infty$,

$$
\operatorname{Pr}\left\{\mathbf{V}_{1}>x\right\} \geq(1+\mathrm{o}(1))(1-\rho) c_{2} \sqrt{2 \pi / c_{3}} \frac{x^{\frac{1}{2} r_{1}}}{(\ln x)^{1-\frac{1}{2} r_{1}}} \mathrm{e}^{-\left(c_{1}+\delta\right)(x \ln x)^{r_{1}}}
$$

where $r_{1}=\eta_{1} /\left(1+\eta_{1}\right)$ and

$$
\begin{aligned}
& c_{0}=\left(1-r_{1}\right)\left(\nu_{2}-1\right) /\left(\eta_{1} \mu_{1}\right), \\
& c_{1}=\mu_{1}\left(c_{0}\right)^{r_{1}}+\left(1-r_{1}\right)\left(\nu_{2}-1\right)\left(1 / c_{0}\right)^{1-r_{1}}, \\
& c_{2}=\left(1 / c_{0}\right)^{2\left(1-r_{1}\right)}, \\
& c_{3}=\mu_{1} \eta_{1}\left(\eta_{1}-1\right)\left(c_{0}\right)^{3 r_{1}-2}+2\left(1-r_{1}\right)\left(\nu_{2}-1\right)\left(1 / c_{0}\right)^{3\left(1-r_{1}\right)} .
\end{aligned}
$$

\section{Proof}

As in the proof of Theorem 3.1, the lower bound relies on the fact that the sojourn time of a class- 1 user will certainly exceed the level $x$ if it finds $k$ other users in the system, each of them, as well as the class-1 user itself, having a remaining service requirement of at least $x /(k+1)$.

Note that for any fixed $\varepsilon>0$ and $x$ large enough, $\operatorname{Pr}\left\{\mathbf{B}_{2}^{r}>x\right\}>x^{1-\nu_{2}-\varepsilon}$. We thus obtain, for $x$ large enough,

$$
\begin{aligned}
& \operatorname{Pr}\left\{\mathbf{V}_{1}>x\right\} \geq \sum_{k=0}^{\infty}(1-\rho) \rho_{2}^{k} \operatorname{Pr}\left\{\mathbf{B}_{1}>\frac{x}{k+1}\right\}\left(\operatorname{Pr}\left\{\mathbf{B}_{2}^{r}>\frac{x}{k+1}\right\}\right)^{k} \\
& \geq \sum_{k=0}^{\infty} \int_{u=k}^{k+1}(1-\rho) \rho_{2}^{u} \operatorname{Pr}\left\{\mathbf{B}_{1}>\frac{x}{u}\right\}\left(\operatorname{Pr}\left\{\mathbf{B}_{2}^{r}>\frac{x}{u}\right\}\right)^{u} \mathrm{~d} u \\
& \geq(1-\rho) \int_{u=0}^{\infty} \mathrm{d} u \mathrm{e}^{u \ln \rho_{2}-\mu_{1}\left(\frac{x}{u}\right)^{\eta_{1}}+u\left(1-\nu_{2}-\varepsilon\right) \ln \frac{x}{u}} \\
& =(1-\rho) \frac{x^{r_{1}}}{(\ln x)^{1-r_{1}}} \int_{y=0}^{\infty} \frac{\mathrm{d} y}{y^{2}} \mathrm{e}^{-(x \ln x)^{r_{1}}\left(-\frac{\ln \rho_{2}}{y \ln x}+\mu_{1} y^{\eta_{1}}-\frac{1-\nu_{2}-\varepsilon}{y \ln x} \ln \left(y(x \ln x)^{1-r_{1}}\right)\right)}
\end{aligned}
$$




$$
\begin{aligned}
& \geq(1-\rho) \frac{x^{r_{1}}}{(\ln x)^{1-r_{1}}} \int_{y=m}^{M} \frac{\mathrm{d} y}{y^{2}} \mathrm{e}^{-(x \ln x)^{r_{1}}}\left(\mu_{1} y^{\eta_{1}}+\frac{\left(1-r_{1}\right)\left(\nu_{2}+\varepsilon-1\right)}{y}+h_{\varepsilon}(x, y)\right) \\
& \geq(1-\rho) \frac{x^{r_{1}} \mathrm{e}^{-(x \ln x)^{r_{1}} h_{\varepsilon, m, M}(x)}}{(\ln x)^{1-r_{1}}} \int_{y=m}^{M} \frac{\mathrm{d} y}{y^{2}} \mathrm{e}^{-(x \ln x)^{r_{1}} f_{\varepsilon}(y)}
\end{aligned}
$$

with $0<m<M<\infty$,

$$
\begin{aligned}
& h_{\varepsilon}(x, y)=\frac{1}{y \ln x}\left(-\ln \rho_{2}+\left(\nu_{2}+\varepsilon-1\right)\left(\ln y+\left(1-r_{1}\right) \ln (\ln x)\right)\right), \\
& h_{\varepsilon, m, M}(x)=\frac{1}{m \ln x}\left(-\ln \rho_{2}+\left(\nu_{2}+\varepsilon-1\right)\left(\ln M+\left(1-r_{1}\right) \ln (\ln x)\right)\right),
\end{aligned}
$$

and

$$
f_{\varepsilon}(y)=\mu_{1} y^{\eta_{1}}+\frac{\left(1-r_{1}\right)\left(\nu_{2}+\varepsilon-1\right)}{y} .
$$

Note that $f_{\varepsilon}(y)$ attains its minimal value for

$$
y=y_{\varepsilon}:=\left(\frac{\left(1-r_{1}\right)\left(\nu_{2}+\varepsilon-1\right)}{\eta_{1} \mu_{1}}\right)^{1-r_{1}} .
$$

Choose $m<y_{\varepsilon}$ and $M>y_{\varepsilon}$. Using the Laplace method [15], we may replace $f_{\varepsilon}(y)$ in the exponent by $f_{\varepsilon}\left(y_{\varepsilon}\right)+\frac{1}{2} f_{\varepsilon}^{\prime \prime}\left(y_{\varepsilon}\right)\left(y-y_{\varepsilon}\right)^{2}$ and $\frac{\mathrm{d} y}{y^{2}}$ by $\frac{\mathrm{d} y}{\left(y_{\varepsilon}\right)^{2}}$ and write, for $x \rightarrow \infty$,

$$
\begin{aligned}
& (1-\rho) \frac{x^{r_{1}} \mathrm{e}^{-(x \ln x)^{r_{1}} h_{\varepsilon, m, M}(x)}}{(\ln x)^{1-r_{1}}} \int_{y=m}^{M} \frac{\mathrm{d} y}{y^{2}} \mathrm{e}^{-(x \ln x)^{r_{1}} f_{\varepsilon}(y)} \\
& \sim(1-\rho) \frac{x^{r_{1}} \mathrm{e}^{-(x \ln x)^{r_{1} h_{\varepsilon, m, M}(x)}}}{(\ln x)^{1-r_{1}}} \int_{y=-\infty}^{\infty} \frac{\mathrm{d} y}{\left(y_{\varepsilon}\right)^{2}} \mathrm{e}^{-(x \ln x)^{r_{1}}\left(f_{\varepsilon}\left(y_{\varepsilon}\right)+\frac{1}{2} f_{\varepsilon}^{\prime \prime}\left(y_{\varepsilon}\right)\left(y-y_{\varepsilon}\right)^{2}\right)} \\
& \sim(1-\rho) \frac{\sqrt{2 \pi} x^{r_{1}}}{\left(y_{\varepsilon}\right)^{2}(\ln x)^{1-r_{1}} \sqrt{(x \ln x)^{r_{1}} f_{\varepsilon}^{\prime \prime}\left(y_{\varepsilon}\right)}} \mathrm{e}^{-(x \ln x)^{r_{1}}\left(f_{\varepsilon}\left(y_{\varepsilon}\right)+h_{\varepsilon, m, M}(x)\right) .}
\end{aligned}
$$

Let $\delta>0$ be arbitrary. For fixed $m<y_{0}$ and $M>y_{0}, \varepsilon$ close enough to 0 and $x$ large enough, we have that $y_{\varepsilon} \in(m, M)$ and $f_{\varepsilon}\left(y_{\varepsilon}\right)+h_{\varepsilon, m, M}(x)<f_{0}\left(y_{0}\right)+\delta$. Thus, for fixed $\delta>0, \varepsilon$ close enough to 0 and $x \rightarrow \infty$, we have

$$
\operatorname{Pr}\left\{\mathbf{V}_{1}>x\right\} \geq \frac{(1+o(1))(1-\rho) \sqrt{2 \pi} x^{r_{1}}}{\left(y_{\varepsilon}\right)^{2}(\ln x)^{1-r_{1}} \sqrt{(x \ln x)^{r_{1}} f_{\varepsilon}^{\prime \prime}\left(y_{\varepsilon}\right)}} \mathrm{e}^{-(x \ln x)^{r_{1}}\left(f_{0}\left(y_{0}\right)+\delta\right)},
$$

and the proof is completed by letting $\varepsilon \rightarrow 0$.

\section{Conclusion}

Theorem 4.1 shows what happens if no admission control is applied: The fact that there can be arbitrarily many heavy-tailed flows causes a dramatic change in the qualitative delay performance of the light-tailed flows, manifesting itself in a markedly heavier tail of the sojourn time distribution. This starkly contrasts with the fact that, when served 
in isolation [3], or when admission control is performed (Section 3 of the present paper), the sojourn time distribution has an exponential tail whenever the service requirement distribution does so. This dichotomy in qualitative behavior illustrates a trade-off between the number of blocked users and the number of users experiencing long transfer delays. In practice, this trade-off may actually not be sharp, since users incurring long delays may abandon due to impatience effects, thus wasting system resources.

\section{A Proof of Lemma 3.2}

By definition,

$$
\begin{aligned}
\operatorname{Pr}\left\{N_{>\epsilon x}(0, x) \geq K\right\} & =\operatorname{Pr}\left\{N_{>\epsilon x}(0)+M_{>\epsilon x}(0, x) \geq K\right\} \\
& =\sum_{k=0}^{K} \operatorname{Pr}\left\{N_{>\epsilon x}(0)=k\right\} \operatorname{Pr}\left\{M_{>\epsilon x}(0, x) \geq K-k\right\} .
\end{aligned}
$$

Using (2), we obtain $\operatorname{Pr}\left\{N_{>\epsilon x}(0)=k\right\}=\mathrm{O}\left(\left(\operatorname{Pr}\left\{\mathbf{B}_{2}^{r}>\epsilon x\right\}\right)^{k}\right)$ as $x \rightarrow \infty$.

Observe that $M_{>\epsilon x}(0, x)$ (representing the total number of admitted users between time 0 and $x$ ) can be bounded from above by a Poisson random variable (the total number of arrivals). Hence,

$$
\begin{aligned}
\operatorname{Pr}\left\{M_{>\epsilon x}(0, x) \geq l\right\} & \leq \mathrm{e}^{-\lambda x \operatorname{Pr}\{\mathbf{B}>\epsilon x\}} \sum_{m=l}^{\infty} \frac{(\lambda x \operatorname{Pr}\{\mathbf{B}>\epsilon x\})^{m}}{m !} \\
& \leq \sum_{m=l}^{\infty}(\lambda x \operatorname{Pr}\{\mathbf{B}>\epsilon x\})^{m}=\frac{(\lambda x \operatorname{Pr}\{\mathbf{B}>\epsilon x\})^{l}}{1-\lambda x \operatorname{Pr}\{\mathbf{B}>\epsilon x\}} \\
& =\mathrm{O}\left(\left(x \operatorname{Pr}\left\{\mathbf{B}_{2}>\epsilon x\right\}\right)^{l}\right)=\mathrm{O}\left(\left(\operatorname{Pr}\left\{\mathbf{B}_{2}^{r}>\epsilon x\right\}\right)^{l}\right) .
\end{aligned}
$$

In the last step we used that $\operatorname{Pr}\left\{\mathbf{B}_{2}>x\right\}$ is regularly varying. We thus have

$$
\operatorname{Pr}\left\{N_{>\epsilon x}(0, x) \geq K\right\}=\mathrm{O}\left(\left(\operatorname{Pr}\left\{\mathbf{B}_{2}^{r}>\epsilon x\right\}\right)^{K}\right)=\mathrm{o}\left(\left(\operatorname{Pr}\left\{\mathbf{B}_{2}^{r}>\epsilon x\right\}\right)^{K-1}\right),
$$

as $x \rightarrow \infty$.

\section{B Proof of Lemma 3.3}

By definition, the event $T(\alpha x,(\alpha+\delta) x) \leq E(x)$ means that except for a period of length at most $E(x)$ there are constantly $K-1$ users other than the tagged user present in the system during the time interval $[\alpha x,(\alpha+\delta) x]$. Combined with $N_{>\epsilon x}(\alpha x,(\alpha+\delta) x) \leq K-2$, this implies two things: (i) The total amount of service received during the interval $[\alpha x,(\alpha+\delta) x]$ by users whose service time is smaller than $\epsilon x$ must be larger than $(\delta x-E(x)) / K$. Letting $M_{\leq \epsilon x}(s, t)$ be the number of new users admitted into the system during the time interval $[s, t]$ whose service requirement is at most $\epsilon x$, we have

$$
\sum_{j=1}^{M_{\leq \epsilon x}(\alpha x,(\alpha+\delta) x)} \mathbf{B}_{j}(\epsilon x) \geq \frac{\delta x-E(x)}{K}-(K-1) \epsilon x,
$$


with $\mathbf{B}_{1}(\epsilon x), \mathbf{B}_{2}(\epsilon x), \ldots$ an i.i.d. sequence of random variables with $\mathbf{B}_{j}(\epsilon x) \stackrel{d}{=} \mathbf{B} \mid \mathbf{B} \leq \epsilon x$. (ii) The sum of the interarrival times of the users which are admitted into the system during the time interval $[\alpha x,(\alpha+\delta) x]$ must be smaller than $(K-1) E(x)$, i.e.,

$$
\sum_{j=1}^{M_{\leq \epsilon x}(\alpha x,(\alpha+\delta) x)} \mathbf{A}_{j} \leq(K-1) E(x),
$$

with $\mathbf{A}_{1}, \mathbf{A}_{2}, \ldots$ a sequence of independent exponentially distributed random variables with parameter $\lambda$. For compactness, denote $N_{>\epsilon x}:=N_{>\epsilon x}(\alpha x,(\alpha+\delta) x)$ and $M_{\leq \epsilon x}:=$ $M_{\leq \epsilon x}(\alpha x,(\alpha+\delta) x)$. The above considerations can then be combined to obtain the upper bound

$$
\begin{aligned}
& \operatorname{Pr}\left\{T(\alpha x,(\alpha+\delta) x) \leq E(x) ; N_{>\epsilon x} \leq K-2\right\} \\
= & \operatorname{Pr}\left\{T(\alpha x,(\alpha+\delta) x) \leq E(x) ; N_{>\epsilon x} \leq K-2 ; M_{\leq \epsilon x} \leq F(x)\right\} \\
+ & \operatorname{Pr}\left\{T(\alpha x,(\alpha+\delta) x) \leq E(x) ; N_{>\epsilon x} \leq K-2 ; M_{\leq \epsilon x}>F(x)\right\} \\
\leq & \operatorname{Pr}\left\{M_{\leq \epsilon x} \leq F(x), \sum_{j=1}^{M_{\leq \epsilon x}} \mathbf{B}_{j}(\epsilon x) \geq(\delta x-E(x)) / K-(K-1) \epsilon x\right\} \\
+ & \operatorname{Pr}\left\{M_{\leq \epsilon x}>F(x), \sum_{j=1}^{M_{\leq \epsilon x}} \mathbf{A}_{j} \leq(K-1) E(x)\right\} \\
\leq & \operatorname{Pr}\left\{\sum_{j=1} \mathbf{B}_{j}(\epsilon x) \geq(\delta x-E(x)) / K-(K-1) \epsilon x\right\}+\operatorname{Pr}\left\{\sum_{j=1}^{F(x)} \mathbf{A}_{j} \leq(K-1) E(x)\right\} .
\end{aligned}
$$

Choose $F(x):=\max \left(\mathrm{e} \lambda K E(x), x^{-\gamma}\right)$, for some $\gamma>0$. Then

$$
\begin{aligned}
\operatorname{Pr}\left\{\sum_{j=1}^{F(x)} \mathbf{A}_{j} \leq(K-1) E(x)\right\} & =\sum_{l=F(x)}^{\infty} \mathrm{e}^{-\lambda(K-1) E(x)} \frac{(\lambda(K-1) E(x))^{l}}{l !} \\
& \leq \frac{(\lambda(K-1) E(x))^{F(x)}}{F(x) !} \\
& \sim \frac{(\lambda(K-1) E(x))^{F(x)}}{F(x)^{F(x)+\frac{1}{2}} \mathrm{e}^{-F(x)} \sqrt{2 \pi}} \\
& \leq\left(\frac{K-1}{K}\right)^{F(x)}=\mathrm{o}\left(x^{-\zeta}\right),
\end{aligned}
$$

and

$$
\begin{aligned}
& \operatorname{Pr}\left\{\sum_{j=1}^{F(x)} \mathbf{B}_{j}(\epsilon x) \geq(\delta x-E(x)) / K-(K-1) \epsilon x\right\} \\
& =\operatorname{Pr}\left\{\sum_{j=1}^{F(x)}\left(\mathbf{B}_{j}(\epsilon x)-\beta-\theta\right) \geq(\delta x-E(x)) / K-(\beta+\theta) F(x)-(K-1) \epsilon x\right\} .
\end{aligned}
$$

Then, using Lemma 2.1 in [17], the latter quantity may be bounded by a regularly varying function of arbitrary index since $E(x)+(\beta+\theta) K F(x) \leq \delta x / 2-K(K-1) \epsilon x$ for $\epsilon>0$ sufficiently small. 


\section{References}

[1] Altman, E., Avrachenkov, K., Barakat, C., Núñez-Queija, R. (2001). State-dependent M/G/1 type queueing analysis for congestion control in data networks. In: Proc. Infocom 2001 Conference, Anchorage AK, USA, 1350-1359.

[2] Ben Fredj, S., Bonald, T., Proutière, A., Régnié, G., Roberts, J.W. (2001). Statistical bandwidth sharing: a study of congestion at the flow level. In: Proc. Sigcomm 2001, $111-122$

[3] Borst, S.C., Boxma, O.J., Morrison, J.A., Núñez-Queija, R. (2003). The equivalence between processor sharing and random order of service. Oper. Res. Lett. 31, 254-262.

[4] Boyer, J., Guillemin, F., Robert, Ph., Zwart, A.P. (2003). Heavy-tailed M/G/1/PS queues with impatience and admission control in packet networks. In: Proc. Infocom 2003 Conference, San Francisco CA.

[5] Cohen, J.W. (1979). The multiple phase service network with generalized processor sharing. Acta Informatica 12, 245-284.

[6] Crovella, M., Bestavros, A. (1996). Self-similarity in World Wide Web traffic: evidence and possible causes. In: Proc. ACM Sigmetrics '96, 160-169.

[7] Flatto, L. (1997). The waiting time distribution for the random order service M/M/1 queue. Ann. Appl. Prob. 7, 382-409.

[8] Guillemin, F., Robert, Ph., Zwart, A.P. (2002). Performance of TCP in the presence of correlated packet losses. In: Proc. 15th ITC Specialist Seminar.

[9] Jelenković, P.R., Momčilović, P. (2003). Large deviation analysis of subexponential waiting times in a Processor-Sharing queue. Math. Oper. Res. 28, 587-608.

[10] Kelly, F.P. (1979). Reversibility and Stochastic Networks. Wiley, Chichester.

[11] Massoulié, L., Roberts, J.W. (1999). Arguments in favor of admission control for TCP flows. In: Teletraffic Engineering in a Competitive World, Proc. ITC-16, Edinburgh, UK, eds. P. Key, D. Smith (North-Holland, Amsterdam), 33-44.

[12] Massoulié, L., Roberts, J.W. (1999). Bandwidth sharing: Objectives and algorithms. In: Proc. Infocom 1999 Conference, New York NY, USA, 1395-1403.

[13] Núñez-Queija, R. (2000). Processor-Sharing Models for Integrated-Services Networks. Ph.D. Thesis, Eindhoven University of Technology, ISBN 90-646-4667-8.

[14] Núñez-Queija, R. (2002). Queues with equally heavy sojourn time and service requirement. Ann. Oper. Res. 113, 101-117.

[15] Olver, F.W.J. (1974). Asymptotics and Special Functions. Academic Press, New York.

[16] Padhye, J., Firoiu, V., Towsley, D., Kurose, J. (1998). Modeling TCP throughput: a simple model and its empirical validation. In: ACM Comp. Commun. Rev. 28, Proc. ACM Sigcomm '98, 303-314. 
[17] Resnick, S., Samorodnitsky, G. (1999). Activity periods of an infinite server queue and performance of certain heavy tailed fluid queues. Queueing Systems 33, 43-71.

[18] Zwart, A.P. (1999). Sojourn times in a multiclass processor sharing queue. In: Teletraffic Engineering in a Competitive World, Proc. ITC-16, Edinburgh, UK, eds. P. Key, D. Smith (North-Holland, Amsterdam), 335-344.

[19] Zwart, A.P., Boxma, O.J. (2000). Sojourn time asymptotics in the M/G/1 processor sharing queue. Queueing Systems 35, 141-166. 